\title{
One-dimensional nature of protein low-energy vibrations
}

\author{
Minhao Yu, ${ }^{1}$ Pan Tan $\odot,{ }^{1,2}$ Yiyang Ye, ${ }^{3}$ David J. Voneshen, ${ }^{4,5}$ Xiangjun Xing, ${ }^{1,6,7,}{ }^{*}$ and Liang Hong $\oplus^{1,2, \dagger}$ \\ ${ }^{1}$ School of Physics and Astronomy, Shanghai Jiao Tong University, Shanghai 200240, China \\ ${ }^{2}$ Institute of Natural Sciences, Shanghai Jiao Tong University, Shanghai 200240, China \\ ${ }^{3}$ Zhiyuan College, Shanghai Jiao Tong University, Shanghai 200240, China \\ ${ }^{4}$ ISIS Pulsed Neutron and Muon Source, Rutherford Appleton Laboratory, Chilton, Didcot OX11 OQX, United Kingdom \\ ${ }^{5}$ Department of Physics, Royal Holloway University of London, Egham TW20 OEX, United Kingdom \\ ${ }^{6}$ T. D. Lee Institute, Shanghai Jiao Tong University, Shanghai 200240, China \\ ${ }^{7}$ Wilczek Quantum Center, Shanghai Jiao Tong University, Shanghai 200240, China
}

(Received 28 March 2020; accepted 23 July 2020; published 27 August 2020)

\begin{abstract}
Protein internal dynamics is crucial for its function. In particular, low-energy vibrational modes at 1-10 meV play important roles in the transportation of energy inside the protein molecule, and facilitate its enzymatic function and binding to ligands and other biomolecules. However, the microscopic spatiotemporal details of these modes have remained largely unknown, due to limitations of the experimental techniques. Here, by applying inelastic neutron scattering on a perdeuterated protein, we demonstrate that these vibration modes are correlated primarily through peptide bonds rather than noncovalent interactions (including hydrogen bonds), which is further confirmed by a complementary molecular dynamics simulation. More importantly, the complex spatiotemporal features of interatomic vibrations observed in an all-atom simulation are qualitatively reproduced by an ultrasimple toy model, a one-dimensional harmonic chain, where the vibrations propagate along the peptide chain, but are confined and damped by noncovalent interactions surrounding the chain. Our findings are fundamentally important for understanding many functional processes in proteins that are strongly coupled to low-energy vibrational modes. Moreover, the one-dimensional nature of low-energy vibrations discovered here should be applicable to other biomacromolecules and many ordinary polymeric materials.
\end{abstract}

DOI: 10.1103/PhysRevResearch.2.032050

\section{INTRODUCTION}

Protein vibrations span a wide energy window, from high-energy ( $\sim 1000 \mathrm{meV}$, femtosecond) local bond stretching to low-energy $(\sim 1 \mathrm{meV}$, picosecond) collective modes $[1,2]$. Low-energy collective vibrations are crucial for the transportation of thermal energy and perturbation in protein molecules [3,4]. An example is transportation of perturbation starting from hydration water on the protein surface to amino acids deep inside the biomolecule, which impacts its enzymatic function [4]. These modes also play an important role in the lowering of energy barriers and speeding up the catalytic reactions [5] of protein molecules, and facilitating large-scale conformational movements [6], as well as binding to ligands and other biomolecule [7,8], etc.

Many experimental techniques, including inelastic incoherent neutron scattering, inelastic x-ray scattering, light scattering, and terahertz spectroscopy, have been applied to study these low-energy modes in proteins [7,9-12]. Previous studies primarily focused on the characteristic frequency

\footnotetext{
*xxing@sjtu.edu.cn

†hongl3liang@sjtu.edu.cn
}

Published by the American Physical Society under the terms of the Creative Commons Attribution 4.0 International license. Further distribution of this work must maintain attribution to the author(s) and the published article's title, journal citation, and DOI. and damping amplitude of these modes, as well as their dependence on temperature, ligand binding, and biomolecular structures [7,9-12]. Additionally, numerical and theoretical studies, e.g., normal mode analysis and elastic network model, indicated that these modes exhibit high cooperativity [13-17]. Despite this progress, the microscopic picture of propagation and dissipation of these low-energy modes has remained elusive.

Coherent neutron scattering directly probes interatomic dynamic correlations [18-20], and therefore constitutes a valuable experimental approach to the low-energy vibration of proteins. Nonetheless, neutron experiments, performed on ordinary proteins, which are full of hydrogen atoms, measure predominantly the self-motions of hydrogen atoms [7,9] due to their ultralarge incoherent scattering cross sections, and thus obscure interatomic correlations. Here, to characterize interatomic vibrations, we applied inelastic neutron scattering on fully deuterated proteins, which has been rarely conducted [20]. Our experiment reveals that the vibrations in an energy window of $1-10 \mathrm{meV}$ are primarily correlated through peptide bonds rather than a secondary structural motif linked by hydrogen bonds. A complementary molecular dynamics (MD) simulation confirmed these experimental findings. More importantly, we found that a toy model of a one-dimensional harmonic chain confined and damped by neighboring residues can fully reproduce the complex spatiotemporal features of interatomic vibrations observed in the all-atom simulation. Our study therefore demonstrates the one-dimensional nature of low-energy vibrations in proteins. 

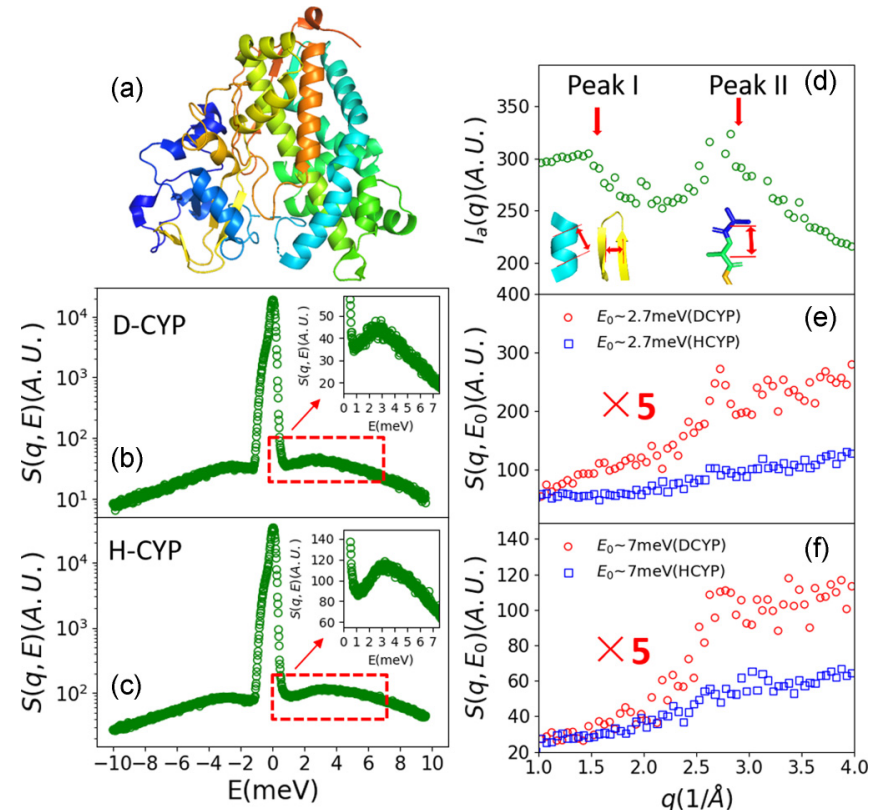

FIG. 1. Experimental dynamic structure factor (DSF) of CYP101. (a) A representative structure of CYP101. DSF of (b) D-CYP and (c) H-CYP measured at $120 \mathrm{~K}$, where the results were summed up in the $q$ range from 1 to $4 \AA^{-1}$ to improve the statistics. As the present work primarily concerns about how vibrations propagate and dissipate inside the protein molecule, we focus the analysis of neutron spectra above $1 \AA^{-1}$ to exclude the contribution of the interprotein scattering at low $q$ to the neutron signals [see the discussion of inter- and intraprotein scattering and Figs. S2(a) and S2(b) in SM [22]]. (d) The approximate static structure factor (SSF) of D-CYP by integrating DSF measured at $5 \mathrm{~K}$ over -10 to $10 \mathrm{meV}$, where peaks I and II are labeled. Comparison of the $q$ dependence of DSF measured on D-CYP and H-CYP at (e) $E_{0}=2.7 \mathrm{meV}$ and (f) $E_{0}=7.0 \mathrm{meV}$. The values of DSF measured on D-CYP were multiplied by a factor of 5 for a better comparison of the shape. The scattering intensities presented in (b)-(f) are relative values by normalizing to the values of a vanadium reference.

\section{NEUTRON EXPERIMENT ON PROTEIN VIBRATIONS}

The neutron scattering experiments were conducted on cytochrome P450 (CYP101). P450's are an important enzyme family catalyzing a variety of biochemical reactions involved in carcinogenesis, drug metabolism, lipid and steroid biosynthesis, and degradation of pollutants in higher organisms [21]. The atomic structure of the protein is illustrated in Fig. 1(a). Both hydrogenated CYP101 and its perdeuterated counterpart were measured using time-of-flight neutron spectroscopy (LET) at ISIS, UK. These two samples are referred to as H-CYP and D-CYP, respectively. Detailed experimental procedures are supplied in the Supplemental Material (SM) [22]. The experimentally measured quantity is the dynamic structure factor (DSF), $S(q, E)$, which characterizes the density of dynamic modes as a function of energy transfer $E$ and wave vector $q$. As shown in Eqs. (S4) and (S5) in SM [22], DSF is a sum of an incoherent component $S_{\text {inc }}(q, E)$ and a coherent component $S_{\text {coh }}(q, E)$. The neutron signals from the H-protein are primarily incoherent $(\sim 90 \%)$, characterizing self-correlations of hydrogen atoms, whereas the ones from the D-protein are mostly coherent ( $\sim 90 \%)$, measuring mostly interatomic correlations of the protein's heavy atoms [23,24].

As displayed in Figs. 1(b) and 1(c) and the corresponding insets, DSF measured on both H-CYP and D-CYP exhibits a prominent inelastic peak around $3 \mathrm{meV}$, which corresponds to a timescale $\sim 1.5 \mathrm{ps}$. The peak is located at $2.7 \mathrm{meV}$ in D-CYP, slightly lower than that in H-CYP (3.1 meV), which might result from the fact that the hydrogen atoms in H-CYP vibrate faster due to their lighter weight as compared to D atoms in D-CYP. The collective nature of a dynamic mode can be determined by analyzing the $q$ dependence of the intensity of the coherent DSF [18-20,23,25]. An in-phase, collective mode at a given energy $E_{0}$ gives $S_{\text {coh }}\left(q, E_{0}\right) \sim$ $I(q) q^{2}$, whereas an uncorrelated mode gives $S_{\text {coh }}\left(q, E_{0}\right) \sim q^{2}$ [18-20,23,25]. Here, $I(q)$ is the static structure factor, providing the spatial correlation between atoms. In the present study, we integrated the dynamical structure factor measured on D-CYP at $5 \mathrm{~K}$ over the energy window from -10 to $10 \mathrm{meV}$, and used its $q$ dependence to approximate the experimental static structure factor, denoted as $I_{a}(q)$. This approximation should work reasonably well as such a low temperature will dramatically suppress the high-energy modes beyond $10 \mathrm{meV}$, which is further supported by the simulation results [see Fig. $\mathrm{S} 2(\mathrm{c})]$. As shown in Fig. 1(d), the so-obtained $I_{a}(q)$ contains two structural peaks, located at $1.4 \AA^{-1}$ (peak I) and $2.7 \AA^{-1}$ (peak II), respectively. Peak I corresponds to a length scale of 4-6 $\AA$, which is the typical distance of secondary structural motifs in the protein. For example, the pitch of $\alpha$-helix is about $5.4 \AA$ and the inter- $\beta$-band distance is $\sim 5 \AA[10,26]$. These secondary structural motifs are formed due to specific inter-residue hydrogen bonds. Peak II corresponds to 2-4 $\AA$, which is the typical distance between two neighboring residues linked by a peptide bond. In Fig. S1, we further show that peak II of the calculated $S(q)$ diminishes drastically when removing every second residue along the peptide chain, while peak I remain almost intact. This verifies that peak II results from correlations between nearest neighbors along the peptide chain.

As shown in Figs. 1(e) and 1(f), at both 2.7 and $7.0 \mathrm{meV}$, the DSF measured on D-CYP, which is dominated by coherent scattering, exhibits a pronounced hump at $\sim 2.7 \AA^{-1}$ (peak II), but not at $1.4 \AA^{-1}$ (peak I). This indicates that the vibration modes in this energy range are primarily correlated through peptide bonds rather than the secondary structural motifs linked by hydrogen bonds. It is consistent with the result of Ref. [27], which demonstrated that the propagation of external thermal perturbation in a peptide helix is primarily through the peptide bond instead of the inter-residue hydrogen bonds. Our finding also agrees with that of a recent neutron scattering experiment on perdeuterated green fluorescent protein [20], which also reported the low cooperativity of low-energy vibrations at peak I. Note, however, Ref. [20] did not probe $q$ above $2 \AA^{-1}$, and hence did not see the strongly correlated vibrations at peak II [Figs. 1(e) and 1(f)].

As a control, we also analyzed the $q$ dependence of DSF measured on H-CYP at both 2.7 and $7 \mathrm{meV}$, which is dominated by incoherent scattering. As shown in Figs. 1(e) and 1(f), it increases roughly monotonically with $q$, with no visible peak in the range. This is expected for self-atomic correlations $[18,20,25]$. 

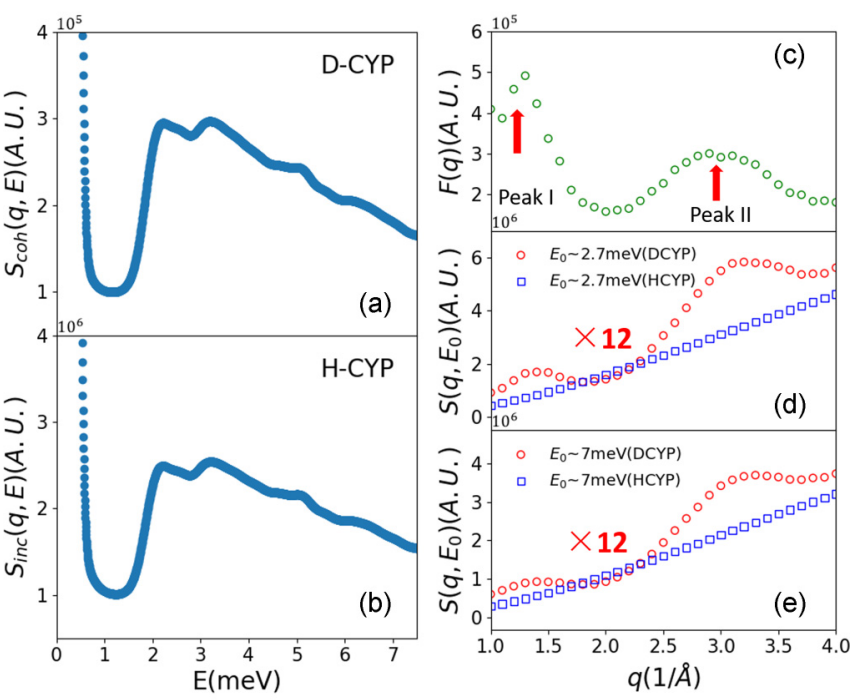

FIG. 2. MD-derived neutron spectra. (a) $S_{\text {coh }}(q, E)$ and (b) $S_{\text {inc }}(q, E)$ at $120 \mathrm{~K}$, where the results were summed up in the $q$ range from 1 to $4 \AA^{-1}$, being consistent with the experiment. (c) The static structure factor of D-CYP calculated from a single protein molecule using the crystal structure. $q$ dependence of the (d) $S_{\text {coh }}\left(q, E_{0}\right)$ and (e) $S_{\text {inc }}\left(q, E_{0}\right)$ at 2.7 and $7.0 \mathrm{meV}$. The calculated $S_{\text {coh }}\left(q, E_{0}=7 \mathrm{meV}\right)$ is multiplied by a factor of 12 for a better comparison of the shape.

\section{MD-DERIVED NEUTRON SPECTRA}

The neutron spectra calculated from the complementary molecular dynamics (MD) simulations were presented in Fig. 2. Detailed MD protocols are supplied in the Supplemental Material [22]. As evident by Figs. 2(a) and 2(b), both the incoherent and coherent DSFs show prominent inelastic peaks around 2-4 meV. $S(q)$ [Fig. 2(c)] also contains peaks I and II. The $q$ dependence of the coherent $\operatorname{DSF} S_{\text {coh }}\left(q, E_{0}\right)$ at fixed $E_{0}(2.7$ and $7.0 \mathrm{meV})$ presents a pronounced peak at $3 \AA^{-1}$ (peak II) alongside a tiny hump at around $1.4 \AA^{-1}$ (peak I) [Figs. 2(d) and 2(e)], implying that these vibrational modes are correlated mostly through peptide bonds. In contrast, the incoherent DSF varies monotonically with $q$ [see Figs. 2(d) and 2(e)]. Hence, the MD-derived neutron spectra (Fig. 2), both dynamical and static structure factors, are in qualitative agreement with the experimental ones (Fig. 1).

\section{TWO-PARTICLE DISPLACEMENT CORRELATION FUNCTION DERIVED FROM MD}

To quantify the spatiotemporal features of protein vibration modes, we use MD trajectories to compute the two-particle displacement correlation function (TPDC),

$$
C_{a b}(t)=\left\langle\left[\vec{r}_{a}\left(t_{0}+t\right)-\vec{r}_{a}\left(t_{0}\right)\right] \cdot\left[\vec{r}_{b}\left(t_{0}+t\right)-\vec{r}_{b}\left(t_{0}\right)\right]\right\rangle,
$$

where $a$ and $b$ label residues, $\vec{r}_{a}\left(t+t_{0}\right)$ and $\overrightarrow{r_{a}}\left(t_{0}\right)$ are the residue positions at time $t+t_{0}$ and $t_{0}$, respectively, while \langle\rangle denotes the time average over the choice of $t_{0}$. TPDC takes a large value if two residues are highly correlated, and becomes zero if they move independently [28,29]. In Fig. 3(a), we compared TPDC at $t^{*}=1.5 \mathrm{ps}$ calculated for three types of residue pairs, which are respectively linked by
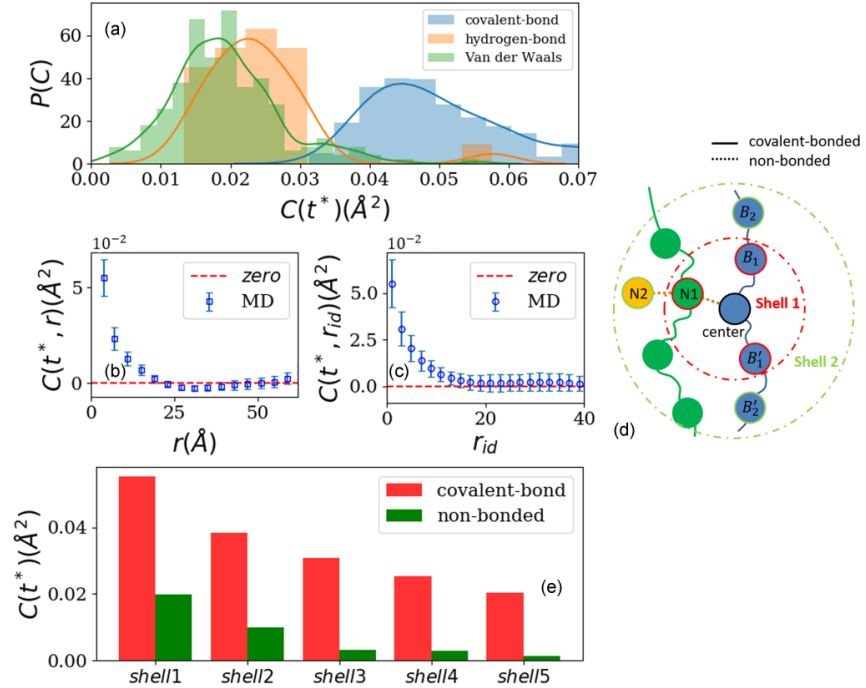

FIG. 3. MD-derived $C\left(t^{*}\right)$ with $t=1.5 \mathrm{ps}$. (a) Histogram of $C\left(t^{*}\right)$ between pairs of residues connected, respectively, by a peptide bond, by a hydrogen bond (HB), and by ordinary van der Waals (vDW) interactions within a distance of $5.5 \AA$. (b) $C\left(t^{*}\right)$ as function of spatial distance $r$. (c) $C\left(t^{*}\right)$ as a function of "chemical distance" $r_{i d}$. To improve the statistics, an ensemble average is performed over all residue pairs with the same $r$ and $r_{i d}$ in (b) and (c), respectively. (d) Schematic diagram for coordination shells (dashed circles). Detailed algorithm for defining coordination shells is provided in SM [22]. (e) $C\left(t^{*}\right)$ as a function of shell numbers, for peptide-bonded neighbors (red) and nonbonded neighbors (green), respectively.

peptide bonds, hydrogen bonds, and ordinary van der Waals interactions within a distance of $5.5 \AA$. The time $t^{*}=1.5 \mathrm{ps}$ corresponds to the inelastic peaks in DSF [Figs. 1(b) and 1(c)]. As illustrated in Fig. 3(a), the peptide-bonded residue pairs show much stronger correlations than the other two types of pairs. This confirms the preceding analysis of neutron scattering profiles [Figs. 1(e), 1(f), 2(d), and 2(e)].

To explore more microscopic details of correlated vibrations, we analyze TPDC as a function of distance between two residues. As shown in Fig. 3(b), $C\left(t^{*}\right)$ decreases monotonically and reaches 0 around $r=20 \AA$, which is close to the radius of the gyration of protein $(21.4 \AA)$. We also analyzed the dependence of TPDC on the "chemical distance," which is defined as the difference between the sequence numbers of two residues, e.g., $r_{i d}=4$ when the two are linked along the peptide chain by three intermediate residues. As seen in Fig. 3(c), $C\left(t^{*}\right)$ decreases monotonically and reaches 0 around $r_{i d}=18$, implying that correlation proceeds along the backbone for nearly 20 residues.

To further distinguish the roles played by peptide bonds and noncovalent interactions, we computed $C\left(t^{*}\right)$ as a function of coordination shell numbers, separately for neighbors connected by peptide bonds and for neighbors connected by noncovalent interactions [see the illustration in Fig. 3(d)]. The obtained results, displayed in Fig. 3(e), again demonstrate that correlations decay much slower for neighbors connected by peptide bonds. Hence it is primarily the peptide chain that propagates the correlated vibrations. 
(a)
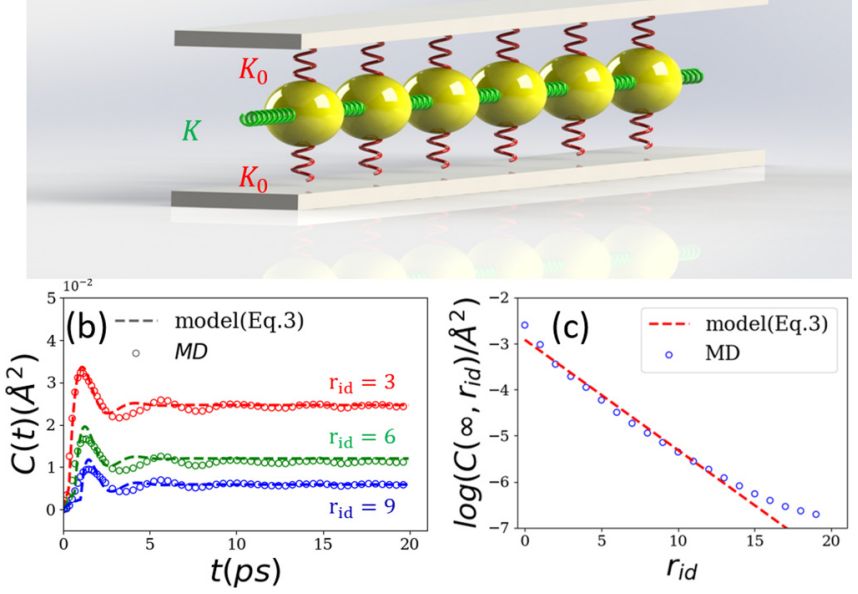

FIG. 4. (a) Illustration of the toy model: One-dimensional harmonic chain confined and damped by neighboring residues. (b) $C(t)$ as a function of $t$ at fixed values of $r_{i d}$. Open symbols correspond to the values calculated from MD, while the dashed lines denote fits using Eq. (3) with a single set of parameters of $K, K_{0}, \lambda$, and $m$. (c) The long-time plateau $C(\infty)$ derived from MD and from Eq. (3). The MD-derived $C(\infty)$ is approximated by averaging $C(t)$ from 15 to 20 ps in (b).

To explore the temporal features of interatomic vibrations along the peptide chain, we analyzed $C(t)$ as a function of $t$ at fixed values of $r_{i d}$. As shown in Fig. 4(b), $C(t)$ oscillates with $t$ similar to an underdamped harmonic oscillator, and converge to a nonzero plateau $C(\infty)$ in the long-time limit.

\section{TOY MODEL: CONFINED UNDERDAMPED HARMONIC CHAIN}

Both our experimental and MD results indicate that protein vibrations on $1-10 \mathrm{meV}$ propagate predominantly along the protein backbone. This discovery prompts us to model protein as a one-dimensional array of beads (residues) connected by springs (peptide bonds), and at the time confined and damped by neighboring residues through the noncovalent interactions [see Fig. 4(a)]. The dynamics of this model can be described by a linear Langevin equation,

$$
\begin{aligned}
& m \ddot{u}_{i}(t)+\lambda \dot{u}_{i}(t)-K\left[u_{i+1}(t)+u_{i-1}(t)-2 u_{i}(t)\right]+K_{0} u_{i}(t) \\
& \quad=\zeta_{i}(t),
\end{aligned}
$$

where $u_{i}(t)$ is the displacement of the $i$ th bead from equilibrium, $m$ the bead mass, and $K$ the strength of peptide bonds. $\lambda$ and $K_{0}$ are the friction coefficient and restoring force coefficient, both due to the confinement of neighboring residues, and finally $\zeta_{i}(t)$ is the white noise, whose variance is related to $\lambda$ via the fluctuation dissipation theorem $\left\langle\zeta_{i}(t) \zeta_{j}(t)\right\rangle=2 k_{B} T \lambda \delta_{i j} \delta\left(t_{i}-t_{j}\right)$. Note that the latter three terms, i.e., friction, restoring, and thermal noise, are all due to noncovalent interactions.
TPDC of this model can be calculated analytically. With details relegated to SM [22], we find

$$
\begin{aligned}
C(t)= & \frac{k_{B} T}{\sqrt{K K_{0}}} e^{-\sqrt{\frac{K_{0}}{K}}} r_{i d}-\frac{k_{B} T}{2 \pi m} e^{-\omega_{c} t} \\
& \times \int_{-\infty}^{+\infty} \frac{\left[\Omega(y)-i \omega_{c}\right] e^{i \Omega(y) t}+\left[\Omega(y)+i \omega_{c}\right] e^{-i \Omega(y) t}}{\Omega(y)\left[\Omega(y)^{2}+\omega_{c}^{2}\right]} \\
& \times e^{i y r_{i d}} d y
\end{aligned}
$$

where $\omega_{c}=\frac{\lambda}{2 m}$ and $\Omega(y)=\sqrt{\frac{y^{2} K}{m}+\frac{K_{0}}{m}-\frac{\lambda^{2}}{4 m^{2}}}$. By fitting Eq. (3) to MD-derived TPDC at different $r_{i d}$ [see Fig. 4(b)], we obtained the best-fitting parameters $m \sim 103 \mathrm{~g} / \mathrm{mol}, K_{0} \sim$ $0.78 \mathrm{~N} / \mathrm{m}, K \sim 13.7 \mathrm{~N} / \mathrm{m}$, and $\lambda \sim 2.7 \times 10^{-13} \mathrm{~kg} / \mathrm{s}$. Note that $K_{0}$ is smaller than $K$ by $\sim 20$ times, quantitatively confirming the result of the elastic network model [17], which assumes distinct force constants for the peptide-bonded and nonbonded residue pairs in the protein. The huge difference between $K_{0}$ and $K$ also naturally explains why vibrations propagate primarily along the backbone [cf. Fig. 3(e)]. Moreover, the best-fitting values of $\lambda$ and $m$ agree quantitatively with the protein internal friction coefficient $\left(\lambda \sim 2 \times 10^{-13} \mathrm{~kg} / \mathrm{s}\right)$ $\left(\lambda \sim 2 \times 10^{-13} \mathrm{~kg} / \mathrm{s}\right.$ reported in Ref. [30], and the average mass of one protein residue $(114 \mathrm{~g} / \mathrm{mol})$, respectively.

As shown in Fig. 4(b), Eq. (3) provides a qualitatively good fitting to MD-computed TPDC at all values of $r_{i d}$ with a single set of parameters. This is remarkable, given the simplicity of this model. With a careful examination of Fig. 4(c), one can see some quantitative difference between the fit and the MD-derived results, particularly at the second oscillation peak around 5 ps. This could result from the fact that the model applied here is extremely abstract without taking into account the complex chemical and structural heterogeneity inside the protein. Such heterogeneity can furnish a distribution of $K$ and $K_{0}$, which could lead to the quantitative difference observed. This might be improved if one replaces the harmonic-chain potential by one or a few low-frequency harmonic modes derived from the normal mode analysis [13,31]. This is, however, beyond the scope of the present work.

Moreover, Eq. (3) predicts the long-time limit of TPDC as $C(\infty)=\frac{k_{B} T}{\sqrt{K K_{0}}} \exp \left(-\sqrt{\frac{K_{0}}{K}} r_{i d}\right)$. Substituting $T=120 \mathrm{~K}$ as well as best-fitting values for $K_{0}$ and $K$, we can calculate $C(\infty)$ as a function of $r_{i d}$. As shown in Fig. 4(c), the obtained results quantitatively agree with those directly derived from MD for $r_{i d}$ between 1 and 15, further validating the toy model [Eq. (3)]. As $C(\infty)=\frac{k_{B} T}{\sqrt{K K_{0}}} \exp \left(-\sqrt{\frac{K_{0}}{K}} r_{i d}\right)$, the characteristic distance for the vibrations to propagate along the peptide chain scales as $\sqrt{\frac{K}{K_{0}}}$. Hence, the bonded interactions are responsible for propagation of the vibrations, while the nonbonded ones dissipate the modes.

\section{CONCLUSION}

In this Rapid Communication, we have performed neutron scattering on fully deuterated cytochrome P450, and studied the interatomic vibrations on $1-10 \mathrm{meV}$. We found that these low-energy vibrations are correlated primarily through peptide bonds rather than nonbonded interactions. More 
importantly, we have shown that the spatiotemporal features of collective vibrations in the protein seen in the all-atom MD simulation can be successfully reproduced by a simple toy model of a confined and underdamped harmonic chain. Our results demonstrate that the low-energy vibrations propagate one dimensionally along the peptide chain, but are damped by the surrounding noncovalent environment. The one-dimensional behaviors of the low-energy vibrations discovered here in the protein are likely to be generally applicable to other biomacromolecules (DNA, RNA, lipids, etc.) or even ordinary linear polymers. Indeed, as shown by Ref. [32], the vibrations in DNA fibers can be interpreted as sound waves through a one-dimensional, monoparticle (basepair) chain along the axis of the DNA fiber.

Collective vibrations are believed to play important roles in the transportation of energy, perturbation, and allosteric large-scale conformational changes in biomacromolecules. Our findings provide crucial insight to a proper understanding of these processes. For example, when a protein molecule binds to a ligand or cofactor or is perturbed by other external sources, the resulting energy will be coupled to the low-energy vibrational modes to proceed through the peptide chain. The large force constant of the peptide bond can ensure a fast transportation of the perturbation, while the dissipation by nonbonded neighbors will slowly distribute the energy to the entire protein molecule.

\section{ACKNOWLEDGMENTS}

The authors acknowledge NSFC Grants No. 11974239, No. 31630002, and No. 11674217, and the Innovation Program of Shanghai Municipal Education Commission. The authors acknowledge the Center for High Performance Computing at Shanghai Jiao Tong University for computing resources, the student innovation center at Shanghai Jiao Tong University, and the support by Shanghai Jiaotong university Multidisciplinary research fund of medicine and engineering (Grant No. YG 2016QN13). X.X. also acknowledges additional support from a Shanghai Talent Program. Experiments at the ISIS Pulsed Neutron and Muon Source were supported by a beamtime allocation from the Science and Technology Facilities Council (RB1690238).
[1] S. Hay and N. S. Scrutton, Good vibrations in enzyme-catalysed reactions, Nat. Chem. 4, 161 (2012).

[2] J. C. Smith, P. Tan, L. Petridis, and L. Hong, Dynamic neutron scattering by biological systems, Annu. Rev. Biophys. 47, 335 (2018).

[3] D. M. Leitner, Energy flow in proteins, Annu. Rev. Phys. Chem. 59, 233 (2008).

[4] P. K. Agarwal, Role of protein dynamics in reaction rate enhancement by enzymes, J. Am. Chem. Soc. 127, 15248 (2005).

[5] S. D. Schwartz and V. L. Schramm, Enzymatic transition states and dynamic motion in barrier crossing, Nat. Chem. Biol. 5, 551 (2009).

[6] V. Fodera, S. Pagliara, O. Otto, U. F. Keyser, and A. M. Donald, Microfluidics reveals a flow-induced large-scale polymorphism of protein aggregates, J. Phys. Chem. Lett. 3, 2803 (2012).

[7] E. Balog, T. Becker, M. Oettl, R. Lechner, R. Daniel, J. Finney, and J. C. Smith, Direct Determination of Vibrational Density of States Change on Ligand Binding to a Protein, Phys. Rev. Lett. 93, 028103 (2004).

[8] B. Tidor and M. Karplus, The contribution of vibrational entropy to molecular association: the dimerization of insulin, J. Mol. Biol. 238, 405 (1994).

[9] L. Hong, D. C. Glass, J. D. Nickels, S. Perticaroli, Z. Yi, M. Tyagi, H. O'Neill, Q. Zhang, A. P. Sokolov, and J. C. Smith, Elastic and Conformational Softness of a Globular Protein, Phys. Rev. Lett. 110, 028104 (2013).

[10] D. Liu, X. Q. Chu, M. Lagi, Y. Zhang, E. Fratini, P. Baglioni, A. Alatas, A. Said, E. Alp, and S. H. Chen, Studies of Phononlike Low-Energy Excitations of Protein Molecules by Inelastic XRay Scattering, Phys. Rev. Lett. 101, 135501 (2008).

[11] G. Acbas, K. A. Niessen, E. H. Snell, and A. G. Markelz, Optical measurements of long-range protein vibrations, Nat. Commun. 5, 3076 (2014).

[12] D. A. Turton, H. M. Senn, T. Harwood, A. J. Lapthorn, E. M. Ellis, and K. Wynne, Terahertz underdamped vibrational motion governs protein-ligand binding in solution, Nat. Commun. 5, 3999 (2014).

[13] V. Kurkal-Siebert and J. C. Smith, Low-temperature protein dynamics: A simulation analysis of interprotein vibrations and the boson peak at $150 \mathrm{~K}, \mathrm{~J}$. Am. Chem. Soc. 128, 2356 (2006)

[14] K. Hinsen, A. J. Petrescu, S. Dellerue, M. C. Bellissent-Funel, and G. R. Kneller, Harmonicity in slow protein dynamics, Chem. Phys. 261, 25 (2000).

[15] I. Bahar and A. J. Rader, Coarse-grained normal mode analysis in structural biology, Curr. Opin. Struct. Biol. 15, 586 (2005).

[16] B. Brooks and M. Karplus, Normal modes for specific motions of macromolecules: application to the hinge-bending mode of lysozyme, Proc. Natl. Acad. Sci. USA 82, 4995 (1985).

[17] D. Ming and M. E. Wall, Allostery in a Coarse-Grained Model of Protein Dynamics, Phys. Rev. Lett. 95, 198103 (2005).

[18] U. Buchenau, A. Wischnewski, D. Richter, and B. Frick, Is the Fast Process at the Glass Transition Mainly due to Long Wavelength Excitations? Phys. Rev. Lett. 77, 4035 (1996).

[19] A. P. Sokolov, Vibrations at the boson peak: random- and coherent-phase contributions, J. Phys.: Condens. Matter 11, A213 (1999).

[20] J. D. Nickels, S. Perticaroli, H. O’Neill, Q. Zhang, G. Ehlers, and A. P. Sokolov, Coherent neutron scattering and collective dynamics in the protein, GFP, Biophys. J. 105, P2182 (2013).

[21] S. G. Sligar, Glimpsing the critical intermediate in cytochrome P450 oxidations, Science 330, 924 (2010).

[22] See Supplemental Material at http://link.aps.org/supplemental/ 10.1103/PhysRevResearch.2.032050 for sample preparation, experimental protocols, MD simulation details and the derivation of the model, which includes Refs. [33-45].

[23] L. Hong, N. Jain, X. L. Cheng, A. Bernal, M. Tyagi, and J. C. Smith, Determination of functional collective motions in a protein at atomic resolution using coherent neutron scattering, Sci. Adv. 2, e1600886 (2016). 
[24] Z. Liu, J. Huang, M. Tyagi, H. O’Neill, Q. Zhang, E. Mamontov, N. Jain, Y. Wang, J. Zhang, J. C. Smith, and L. Hong, Dynamical Transition of Collective Motions in Dry Proteins, Phys. Rev. Lett. 119, 048101 (2017).

[25] J. M. Carpenter and C. A. Pelizzari, Inelastic neutron scattering from amorphous solids. I. Calculation of the scattering law for model structures, Phys. Rev. B 12, 2391 (1975).

[26] P. Etchegoin, Glassylike low-frequency dynamics of globular proteins, Phys. Rev. E 58, 845 (1998).

[27] V. Botan, E. H. Backus, R. Pfister, A. Moretto, M. Crisma, C. Toniolo, P. H. Nguyen, G. Stock, and P. Hamm, Energy transport in peptide helices, Proc. Natl. Acad. Sci. USA 104, 12749 (2007).

[28] M. C. Rheinstädter, J. Das, E. J. Flenner, B. Brüning, T. Seydel, and I. Kosztin, Motional Coherence in Fluid Phospholipid Membranes, Phys. Rev. Lett. 101, 248106 (2008).

[29] O. Takeshi, S. Goto, T. Matsumoto, A. Nakahara, and M. Otsuki, Analytical calculation of four-point correlations for a simple model of cages involving numerous particles, Phys. Rev. E 88, 062108 (2013).

[30] K. Moritsugu and J. C. Smith, Langevin model of the temperature and hydration dependence of protein vibrational dynamics, J. Phys. Chem. B 109, 12182 (2005).

[31] E. Balog, D. Perahia, J. C. Smith, and F. Merzel, Vibrational softening of a protein on ligand binding, J. Phys. Chem. B 115, 6811 (2011).

[32] L. van Eijck, F. Merzel, S. Rols, J. Ollivier, V. T. Forsyth, and M. R. Johnson, Direct Determination of the Base-Pair Force Constant of DNA from the Acoustic Phonon Dispersion of the Double Helix, Phys. Rev. Lett. 107, 088102 (2011).

[33] R. I. Bewley, J. W. Taylor, and S. M. Bennington, LET, a cold neutron multi-disk chopper spectrometer at ISIS, Nucl. Instrum. Methods Phys. Res., Sect. A 637, 128 (2011).

[34] O. Arnold, J. C. Bilheux, J. M. Borreguero, A. Buts, S. I. Campbell, L. Chapon, M. Doucet, N. Draper, R. Ferraz Leal, M. A. Gigg, V. E. Lynch, A. Markvardsen, D. J. Mikkelson, R. L. Mikkelson, R. Miller, K. Palmen, P. Parker, G. Passos, T. G. Perring, P. F. Peterson et al., Mantid-Data analysis and visualization package for neutron scattering and $\mu$ SR experiments, Nucl. Instrum. Methods Phys. Res., Sect. A 764, 156 (2014).
[35] M. J. Abraham, T. Murtola, R. Schulz, S. Páll, J. C. Smith, B. Hess, and E. Lindahl, GROMACS: High performance molecular simulations through multi-level parallelism from laptops to supercomputers, SoftwareX 1-2, 19 (2015).

[36] Y. T. Lee, R. F. Wilson, I. Rupniewski, and D. B. Goodin, P450cam visits an open conformation in the absence of substrate, Biochemistry 49, 3412 (2010).

[37] P. Bjelkmar, P. Larsson, M. A. Cuendet, B. Hess, and E. Lindahl, Implementation of the CHARMM force field in GROMACS: Analysis of protein stability effects from correction maps, virtual interaction sites, and water models, J. Chem. Theory Comput. 6, 459 (2010).

[38] H. W. Horn, W. C. Swope, J. W. Pitera, J. D. Madura, T. J. Dick, G. L. Hura, and T. Head-Gordon, Development of an improved four-site water model for biomolecular simulations: TIP4P-Ew, J. Chem. Phys. 120, 9665 (2004).

[39] U. Essmann, L. Perera, M. L. Berkowitz, T. Darden, H. Lee, and L. G. Pedersen, A smooth particle mesh Ewald method, J. Chem. Phys. 103, 8577 (1995).

[40] G. Bussi, D. Donadio, and M. Parrinello, Canonical sampling through velocity rescaling, J. Chem. Phys. 126, 014101 (2007).

[41] M. Parrinello and A. Rahman, Polymorphic transitions in single crystals: A new molecular dynamics method, J. Appl. Phys. 52, 7182 (1981).

[42] V. F. Sears, Neutron scattering lengths and cross sections, Neutron News 3, 26 (1992).

[43] B. Lindner and J. C. Smith, Sassena-X-ray and neutron scattering calculated from molecular dynamics trajectories using massively parallel computers, Comput. Phys. Commun. 183, 1491 (2012).

[44] L. Hong, M. A. Sharp, S. Poblete, R. Biehl, M. Zamponi, N. Szekely, M.-S. Appavou, R. G. Winkler, R. E. Nauss, A. Johs, J. M. Parks, Z. Yi, X. Cheng, L. Liang, M. Ohl, S. M. Miller, D. Richter, G. Gompper, and J. C. Smith, Structure and dynamics of a compact state of a multidomain protein, the mercuric ion reductase, Biophys. J. 107, P393 (2014).

[45] D. Engberg, A. Wischnewski, U. Buchenau, L. Börjesson, A. J. Dianoux, A. P. Sokolov, and L. M. Torell, Sound waves and other modes in the strong glass former $\mathrm{B}_{2} \mathrm{O}_{3}$, Phys. Rev. B 58 , 9087 (1998). 